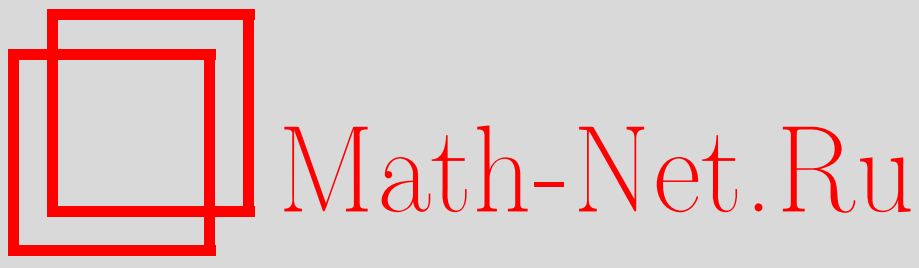

Н. Н. Попов, Л. В. Коваленко, Поля напряжений на границе стохастически неоднородной полуплоскости при ползучести, Вестн. Сам. гос. техн. ун-та. Сер. Физ.мат. науки, 2006, выпуск 42, 61-66

DOI: https://doi.org/10.14498/vsgtu411

Использование Общероссийского математического портала Math-Net.Ru подразумевает, что вы прочитали и согласны с пользовательским соглашением

http://www . mathnet.ru/rus/agreement

Параметры загрузки:

IP: 3.89 .185 .249

26 апреля 2023 г., 05:14:49

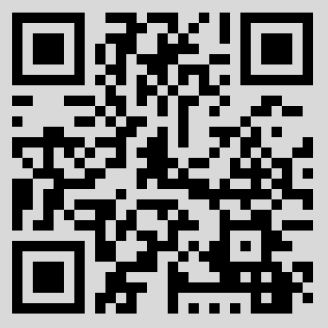


6. Стружанов В.В., Миронов В.И. Деформационное разупрочнение материала в элементах конструкций. — Екатеринбург: Изд-во УрО РАН, 1995. - 192 с.

7. Вильдеман В.Э., Соколкин Ю.В., Ташкинов А.А. Механика неупругого деформирования и разрушения композиционных материалов. - М.: Наука. Физматлит, 1997. - 288 с.

8. Стружанов В.В., Башуров Вяч.В., Каримов П.Ф. К определению параметров инкрементального закона пластичности для изотропных сред // Изв. Урал. гос. ун-та. (сер. Математика и механика. Вып. 2), 1999. — № $14-$ C. $119-134$.

9. Экспериментальные функции сопротивления легированной стали при растяжении и кручении / С.Д. Волков, Ю.П. Гуськов, В.И. Кривоспицкая и др. // Проблемы прочности, 1979. - Т. 11, № 1. - С. 3-6.

10. Работнов Ю.Н. Механика деформируемого твердого тела. - М.: Наука, 1988. — 712 с.

11. Шевченко Ю.Н. К построению поверхности нагружения в теории пластичности // Прикладная механика, 1996. - Т. 32, № 11. - С. 31-37.

12. Седов Л. И. Механика сплошной среды. - М.: Наука, 1970. — Т 2. — 568 с.

13. Райс Д.Р. Об устойчивости дилатантного упрочнения насыщенных скальных массивов // Механика. Новое в зарубежной науке. - Т. 2. Определяющие законы механики грунтов. - М.: Мир, 1975. - С. 195-209.

14. Ильюшин А.А. Об основах общей математической теории пластичности // Вопросы теории пластичности. - М.: Изд-во АН СССР, 1961. - С. 3-29.

15. Ильюшин А.А. Пластичность. - М.: Изд-во АН СССР, 1963. - $271 \mathrm{c}$.

16. Хорн Р., Джснсон Ч. Матричный анализ. - М.: Мир, 1989. - 655 с.

17. Васидзу К. Вариационные методы в теории упругости и пластичности. - М.: Мир, 1987. - 542 с.

18. Писаренко Г.С., Лебедев А.А. Сопротивление материалов деформированию и разрушению при сложном напряженном состоянии. - Киев: Наукова думка, 1969. — 212 с.

Поступила 27.01.2006 г.

УдК 539.376

Н.Н.Попов, Л.В.Коваленко

\section{ПОЛЯ НАПРЯЖЕНИЙ НА ГРАНИЦЕ СТОХАСТИЧЕСКИ НЕОДНОРОДНОЙ ПОЛУПЛОСКОСТИ ПРИ ПОЛЗУЧЕСТИ}

Рассмотрена краевая задача о напряженном состоянии стохастически неоднородной полуплоскости $x_{2} \geq 0$ в условиях ползучести. Определяющее соотношение ползучести, взятое в соответствии с нелинейной теорией вязкого течения, сформулировано в стохастической форме. Поставленная задача решается приближенно относительно компонент тензора напряжений $\sigma_{i j}$ на основе линеаризации по методу малого параметра. Решение линеаризованной задачи получено в виде суммы двух рядов. Первый ряд задает решение вдали от границы полуплоскости без учета краевого эффекта. Члены второго ряда являются функииями координат $x_{2}$, они быстро затухают по мере удаления от гранищь полуплоскости. Произведено исследование концентрации напряжений, возникающей на границе полуплоскости.

Структурная неоднородность материала обусловливает появление ряда механических эффектов, которые не могут быть изучены в рамках классических детерминированных теорий. Одним из таких эффектов является эффект пограничного слоя. Суть его состоит в том, что вблизи границы структурированного тела имеется пограничный слой, обладающий рядом специфических особенностей. В частности, на границе тела возникает концентрация напряжений, которая может достигать заметной величины. Эффект пограничного слоя для упругих тел был исследован в работах [1-5]. В теории ползучести известны решения стохастических задач для внутренних областей, достаточно удаленных от границы тела [6-9], а влияние стохастических неоднородностей на напряженно-деформированное состояние вблизи поверхности, на которой заданы краевые условия, до настоящего времени практически не изучены.

Пусть к границе стохастически неоднородной полуплоскости $x_{2} \geq 0$, находящейся в условиях плоского напряженного состояния, приложены нагрузки

$$
\left.\sigma_{22}\right|_{x_{2}=0}=\sigma_{22}^{0}=\text { const, }\left.\sigma_{12}\right|_{x_{2}=0}=0,
$$

а напряжение $\sigma_{11}$ удовлетворяет условию макроскопической однородности $\left\langle\sigma_{11}\right\rangle=\sigma_{11}^{0}=$ const , которое соответствует приложению при $x_{1}= \pm h$, где $h$ достаточно велико, постоянных по $x_{2}$ напряжений $\sigma_{11}^{0}$. Здесь и далее угловыми скобками обозначена операция математического ожидания.

Определяющее соотношение ползучести принимается в соответствии с нелинейной теорией вязкого течения (установившейся ползучести) в стохастической форме [6] 


$$
\delta_{i j}=c s^{n-1}\left(\sigma_{i j}-\frac{1}{3} \delta_{i j} \sigma_{k k}\right)\left(1+\alpha U\left(x_{1}, x_{2}\right)\right),
$$

где $s$ - интенсивность напряжений:

$$
s^{2}=\frac{1}{2}\left(3 \sigma_{i j} \sigma_{i j}-\sigma_{i i} \sigma_{j j}\right),
$$

$p_{i j}$ — компоненты тензора деформаций ползучести, $\sigma_{i j}$ - компоненты тензора напряжений, $\delta_{i j}$ - символ Кронекера, $U\left(x_{1}, x_{2}\right)$ - случайная однородная функция, описывающая флуктуации реологических свойств материала с математическим ожиданием $\langle U\rangle=0$ и дисперсией $\left\langle U^{2}\right\rangle=1 ; c, n, \alpha-$ постоянные материала, точка означает дифференцирование по времени. По повторяющимся индексам производится суммирование от 1 до 2.

Компоненты тензора напряжений $\sigma_{i j}$ удовлетворяют уравнениям равновесия

$$
\sigma_{i j, j}=0,
$$

а компоненты тензора скоростей деформаций $\alpha_{i j}$ - условиям

$$
\Lambda_{i j} \Lambda_{k l} \&_{j k, i l}=0 \text {, }
$$

которые получены из уравнений совместности деформаций путем дифференцирования по времени. Здесь $\Lambda_{i j}$ - единичный антисимметричный псевдотензор.

Соотношения (2)-(4) при краевых условиях (1) задают стохастическую задачу ползучести, которая в дальнейшем решается приближенно относительно напряжений. Частный случай этой задачи рассматривался в работе [9] при условии $\sigma_{11}^{0}=\sigma_{22}^{0}=\sigma^{0}$, что соответствует равномерному растяжению полуплоскости вдоль главных осей.

Тензор напряжений $\sigma_{i j}$ с учетом (1) может быть представлен в виде суммы двух слагаемых

$$
\sigma_{i j}=\sigma_{i j}^{0}+\sigma_{i j}^{*},\left\langle\sigma_{i j}\right\rangle=\sigma_{i j}^{0},\left\langle\sigma_{i j}^{*}\right\rangle=0,
$$

где $\sigma_{i j}^{*}$ — флуктуации напряжений.

Линеаризация задачи ползучести (2)-(4) была произведена в работе [6]. С целью физической линеаризации функция $s^{n-1}$ была разложена в степенной ряд и в этом разложении были учтены только линейные члены. Для статистической линеаризации определяющего соотношения (2) использовалось корреляционное приближение теории случайных функций, т.е. предполагалось, что произведениями вида $\sigma_{i j}^{*} \sigma_{k l}^{*}, \alpha U \sigma_{i j}^{*}$ допустимо пренебречь. В итоге была получена система линейных дифференциальных уравнений в частных производных относительно флуктуаций напряжений:

$$
\sigma_{i j, j}^{*}=0,
$$

$\sigma_{11,22}^{*}\left(2+k_{1} l_{1}\right)+\sigma_{22,22}^{*}\left(-1+k_{1} l_{2}\right)+\sigma_{11,11}^{*}\left(-1+k_{2} l_{1}\right)+\sigma_{22,11}^{*}\left(2+k_{2} l_{2}\right)-6 \sigma_{12,12}^{*}=-\alpha\left(U_{, 22} l_{1}+U_{, 11} l_{2}\right),(6)$ где $l_{1}=2 \sigma_{11}^{0}-\sigma_{22}^{0}, l_{2}=2 \sigma_{22}^{0}-\sigma_{11}^{0}, k_{i}=\frac{(n-1) l_{i}}{2 s_{0}^{2}}, s_{0}^{2}=\left(\sigma_{11}^{0}\right)^{2}+\left(\sigma_{22}^{0}\right)^{2}-\sigma_{11}^{0} \sigma_{22}^{0}$.

Краевые условия для системы (6) с учетом (1), (5) имеют вид

$$
\left.\sigma_{22}^{*}\right|_{x_{2}=0}=0,\left.\sigma_{12}^{*}\right|_{x_{2}=0}=0 \text {. }
$$

Если ввести функцию напряжений $F$ для флуктуаций тензора напряжений

$$
\sigma_{11}^{*}=F_{, 22}, \sigma_{22}^{*}=F_{, 11}, \sigma_{12}^{*}=-F_{, 12},
$$

то вместо системы уравнений (6) получим единственное дифференциальное уравнение относительно $F$ :

$$
F_{, 1111}\left(2+k_{2} l_{2}\right)+2 F_{, 1122}\left(2+k_{1} l_{2}\right)+F_{, 2222}\left(2+k_{1} l_{1}\right)=-\alpha\left(U_{, 22} l_{1}+U_{, 11} l_{2}\right)
$$

с краевыми условиями

$$
\left.F_{, 11}\right|_{x_{2}=0}=0,\left.F_{, 12}\right|_{x_{2}=0}=0 .
$$

Пусть однородная функция $U\left(x_{1}, x_{2}\right)$, с помощью которой задается случайное поле возмущений реологических свойств материала, является почти периодической быстро осциллирующей функцией [1]

$$
U=\sum_{k=1}^{\infty} A^{(k)} \cos \left(\omega \beta_{s}^{(k)} x_{s}+\phi^{(k)}\right),
$$


где $\omega-$ большой параметр, имеющий размерность, обратную длине; $\beta_{s}^{(k)}-$ безразмерные величины порядка единицы; $A^{(k)}$ — центрированные случайные величины; $\phi^{(k)}-$ случайные величины, распределенные равномерно на участке $(0 ; 2 \pi)$, причем все случайные величины $A^{(k)}$, $\phi^{(k)}$ — независимы. При этом имеем [10]

$$
\left\langle U^{2}\right\rangle=\frac{1}{2} \sum_{k=1}^{\infty} D\left[A^{(k)}\right],
$$

где $D\left[A^{(k)}\right]$ - дисперсия случайной величины $A^{(k)}$. Отсюда с учетом равенства $\left\langle U^{2}\right\rangle=1$ следует, что

$$
\sum_{k=1}^{\infty} D\left[A^{(k)}\right]=2
$$

Для удобства выкладок целесообразно перейти к комплексным функциям

$$
U^{\varrho}=\sum_{k=1}^{\infty} A^{(k)} \exp \left(i \omega \beta_{s}^{(k)} x_{s}\right)
$$

где $\AA^{\left(k k^{k}\right)}=A^{(k)} \exp \left(i \phi^{(k)}\right)$.

Решение краевой задачи (9), (10) можно представить в виде

$$
F=\sum_{k=1}^{\infty}\left(v^{(k)}+w^{(k)}\right),
$$

где $v^{(k)}$ - частное решение уравнения (9), полученное при замене функции $\mathscr{V}^{\mathscr{k}} k$-тым членом разложения (13), а $w^{(k)}$ - решение соответствующего (9) однородного уравнения, удовлетворяющее при $x_{2}=0$ условиям

$$
\left.w_{, 11}^{(k)}\right|_{x_{2}=0}=-\left.v_{, 11}^{(k)}\right|_{x_{2}=0},\left.w_{, 12}^{(k)}\right|_{x_{2}=0}=-\left.v_{, 12}^{(k)}\right|_{x_{2}=0}
$$

В дальнейшем, там, где это не приводит к недоразумениям, верхний индекс будет опускаться.

Функцию $v$ можно искать в виде

$$
v=f \exp \left(i \omega \beta_{s} x_{s}\right), f=\text { const } .
$$

После подстановки (16) в (9) для нахождения $f$ получается алгебраическое уравнение

$$
\omega^{2} \beta_{1}^{4}\left(2+k_{2} l_{2}\right) f+2 \omega^{2} \beta_{1}^{2} \beta_{2}^{2}\left(2+k_{1} l_{2}\right) f+\omega^{2} \beta_{2}^{4}\left(2+k_{1} l_{1}\right) f=\alpha \AA\left(\beta \beta_{2}^{2} l_{1}+\beta_{1}^{2} l_{2}\right),
$$

из которого следует

$$
f=\frac{2 \alpha s_{0}^{2}}{\omega^{2}} \cdot \frac{\AA\left(\rho \beta_{2}^{2} l_{1}+\beta_{1}^{2} l_{2}\right)}{4 s_{0}^{2} \beta^{4}+n\left(\beta_{2}^{2} l_{1}+\beta_{1}^{2} l_{2}\right)^{2}}, \beta^{2}=\beta_{i} \beta_{i} .
$$

Ряд $\sum_{k=1}^{\infty} v^{(k)}$ задает решение задачи вдали от границы полуплоскости без учета краевых эффектов. Функция $w$ имеют характер пограничного слоя: она быстро затухает по мере удаления от границы полуплоскости. Ее можно искать в виде

$$
w=\phi\left(x_{2}\right) \exp \left(i \omega \beta_{1} x_{1}\right),
$$

где функция $\phi\left(x_{2}\right)$ удовлетворяет дифференциальному уравнению

$$
\phi_{, 2222}\left(2+k_{1} l_{1}\right)-2 \omega^{2} \beta_{1}^{2} \phi_{, 22}\left(2+k_{1} l_{2}\right)+\omega^{4} \beta_{1}^{4} \phi\left(2+k_{2} l_{2}\right)=0
$$

с краевыми условиями

$$
\left.\phi\right|_{x_{2}=0}=-f,\left.\phi_{, 2}\right|_{x_{2}=0}=-i \omega \beta_{2} f .
$$

Уравнение (19) получено подстановкой (18) в однородное уравнение, соответствующее (9), а краевые условия получены из условий (15) с использованием выражений (16), (18).

Решение уравнения (19) при $\sigma_{11}^{0} \neq \sigma_{22}^{0}$ имеет вид

$$
\phi=\sum_{s=1}^{4} C_{s} \exp \left(r_{s} x_{2}\right)
$$


где $C_{s}$ - произвольные постоянные, $r_{s}$ - корни характеристического уравнения

$$
\left(2+k_{1} l_{1}\right) r^{4}-2 \omega^{2} \beta_{1}^{2}\left(2+k_{1} l_{2}\right) r^{2}+\omega^{4} \beta_{1}^{4}\left(2+k_{2} l_{2}\right)=0 .
$$

Решение поставленной задачи при $\sigma_{11}^{0}=\sigma_{22}^{0}=\sigma^{0}$ приведено в работе [9].

Корни характеристического уравнения (22) равны

$r_{1,2}=-\omega \beta_{1}\left(\frac{\sqrt{a_{1}+\sqrt{a_{1}^{2}+a_{2}^{2}}}}{\sqrt{2}} \pm i \frac{\sqrt{-a_{1}+\sqrt{a_{1}^{2}+a_{2}^{2}}}}{\sqrt{2}}\right), r_{3,4}=\omega \beta_{1}\left(\frac{\sqrt{a_{1}+\sqrt{a_{1}^{2}+a_{2}^{2}}}}{\sqrt{2}} \pm i \frac{\sqrt{-a_{1}+\sqrt{a_{1}^{2}+a_{2}^{2}}}}{\sqrt{2}}\right)$,

где $a_{1}=\frac{4 s_{0}^{2}+(n-1) l_{1} l_{2}}{4 s_{0}^{2}+(n-1) l_{1}^{2}}, a_{2}=\frac{2 s^{0} \sqrt{n-1}\left|l_{1}-l_{2}\right|}{4 s_{0}^{2}+(n-1) l_{1}^{2}}$.

Из четырех корней характеристического уравнения (22) два корня $r_{3}$ и $r_{4}$ имеют положительные действительные части. Так как при $x \rightarrow \infty$ краевой эффект должен затухать, то две постоянные, соответствующие этим корням, равны нулю. Для нахождения двух других констант $C_{1}$ и $C_{2}$ применяются краевые условия (20). Они равны:

$$
C_{1}=\frac{f\left(r_{2}-i \omega \beta_{2}\right)}{r_{1}-r_{2}}, C_{2}=-\frac{f\left(r_{1}-i \omega \beta_{2}\right)}{r_{1}-r_{2}} .
$$

Решение уравнения (21), бесконечно малое при $x_{1} \rightarrow \infty$, имеет вид

$$
\phi=\frac{f\left(r_{2}-i \omega \beta_{2}\right)}{r_{1}-r_{2}} e^{r_{1} x_{2}}-\frac{f\left(r_{1}-i \omega \beta_{2}\right)}{r_{1}-r_{2}} e^{r_{2} x_{2}} .
$$

Подставляя выражения (16), (18), (23) в (14) можно найти функцию напряжения

$$
F=\sum_{k=1}^{\infty} f^{(k)} \exp \left(i \omega \beta_{1}^{(k)} x_{1}\right)\left[\exp \left(i \omega \beta_{2}^{(k)} x_{2}\right)-\frac{r_{2}^{(k)}-i \omega \beta_{2}^{(k)}}{r_{2}^{(k)}-r_{1}^{(k)}} \exp \left(r_{1}^{(k)} x_{2}\right)+\frac{r_{1}^{(k)}-i \omega \beta_{2}^{(k)}}{r_{2}^{(k)}-r_{1}^{(k)}} \exp \left(r_{2}^{(k)} x_{2}\right)\right] \text {. }
$$

Теперь, используя соотношения (8), решение краевой задачи (6), (7) можно записать в виде

$$
\begin{aligned}
& \sigma_{11}^{*}=\sum_{k=1}^{\infty} f^{(k)} \exp \left(i \omega \beta_{1}^{(k)} x_{1}\right)\left[-\omega^{2}\left(\beta_{2}^{(k)}\right)^{2} \exp \left(i \omega \beta_{2}^{(k)} x_{2}\right)-\frac{\left(r_{1}^{(k)}\right)^{2}\left(r_{2}^{(k)}-i \omega \beta_{2}^{(k)}\right)}{r_{2}^{(k)}-r_{1}^{(k)}} \exp \left(r_{1}^{(k)} x_{2}\right)+\right. \\
& \left.+\frac{\left(r_{2}^{(k)}\right)^{2}\left(r_{1}^{(k)}-i \omega \beta_{2}^{(k)}\right)}{r_{2}^{(k)}-r_{1}^{(k)}} \exp \left(r_{2}^{(k)} x_{2}\right)\right] \\
& \sigma_{22}^{*}=\sum_{k=1}^{\infty} f^{(k)} \omega^{2}\left(\beta_{1}^{(k)}\right)^{2} \exp \left(i \omega \beta_{1}^{(k)} x_{1}\right)\left[-\exp \left(i \omega \beta_{2}^{(k)} x_{2}\right)-\frac{r_{2}^{(k)}-i \omega \beta_{2}^{(k)}}{r_{2}^{(k)}-r_{1}^{(k)}} \exp \left(r_{1}^{(k)} x_{2}\right)+\right. \\
& \left.+\frac{r_{1}^{(k)}-i \omega \beta_{2}^{(k)}}{r_{2}^{(k)}-r_{1}^{(k)}} \exp \left(r_{2}^{(k)} x_{2}\right)\right] \\
& \sigma_{12}^{*}=\sum_{k=1}^{\infty} f^{(k)} \omega \beta_{1}^{(k)} \exp \left(i \omega \beta_{1}^{(k)} x_{1}\right)\left[-\omega \beta_{2}^{(k)} \exp \left(i \omega \beta_{2}^{(k)} x_{2}\right)-\frac{i r_{1}^{(k)}\left(r_{2}^{(k)}-i \omega \beta_{2}^{(k)}\right)}{r_{2}^{(k)}-r_{1}^{(k)}} \exp \left(r_{1}^{(k)} x_{2}\right)+\right. \\
& \left.+\frac{i r_{2}^{(k)}\left(r_{1}^{(k)}-i \omega \beta_{2}^{(k)}\right)}{r_{2}^{(k)}-r_{1}^{(k)}} \exp \left(r_{2}^{(k)} x_{2}\right)\right] \text {. }
\end{aligned}
$$

Используя решение краевой задачи (24), можно найти статистические характеристики случайного поля напряжений в любой точке полуплоскости.

Дальнейшие расчеты будут производиться при условии, что все величины $\beta_{i}^{(k)}$ равны единице. При этом условии случайное поле $U$, заданное разложением (11), можно считать приблизительно изотропным [1]. 
Проведем исследование концентрации напряжений на границе полуплоскости $x_{2}=0$. Согласно граничным условиям (7)

$$
\sigma_{22}^{*}(0)=\left.\sigma_{22}^{*}\right|_{x_{2}=0}=0, \sigma_{12}^{*}(0)=\left.\sigma_{12}^{*}\right|_{x_{2}=0}=0,
$$

а первая из формул (24) при $x_{2}=0$ дает $\left(\beta_{i}^{(k)}=1\right)$ :

$$
\sigma_{11}^{*}(0)=\exp \left(i \omega x_{1}\right)\left(-\omega^{2}+r_{1} r_{2}-i \omega\left(r_{1}+r_{2}\right)\right) \sum_{k=1}^{\infty} f^{(k)},
$$

Последнее выражение после подстановки постоянной $f^{(k)}$ (формула (17)) и корней $r_{1}$ и $r_{2}$ характеристического уравнения (22) преобразуется к виду

$$
\left.\sigma_{11}^{*}(0)=\frac{2 \alpha s_{0}^{2}\left(l_{1}+l_{2}\right)}{16 s_{0}^{2}+(n-1)\left(l_{1}+l_{2}\right)^{2}} \exp \left(i \omega x_{1}\right)\left(-1+\sqrt{a_{1}^{2}+a_{2}^{2}}+i \sqrt{2}\left(\sqrt{a_{1}+\sqrt{a_{1}^{2}+a_{2}^{2}}}\right)\right) \sum_{k=1}^{\infty} \not b^{k}\right) .
$$

Дисперсия напряжения $\sigma_{11}$ на границе полуплоскости $x_{2}=0$ с учетом формулы (12) выражается следующим образом

$$
D_{11}(0)=\left\langle\left.\sigma_{11}^{*}(0)\right|^{2}\right\rangle=\frac{8 \alpha^{2} s_{0}^{4}\left(l_{1}+l_{2}\right)^{2}}{\left(16 s_{0}^{2}+(n-1)\left(l_{1}+l_{2}\right)^{2}\right)^{2}}\left(1+a_{1}^{2}+a_{2}^{2}+2 a_{1}\right),
$$

а при $x \rightarrow \infty$ она равна

$$
D_{11}(\infty)=\frac{8 \alpha^{2} s_{0}^{4}\left(l_{1}+l_{2}\right)^{2}}{\left(16 s_{0}^{2}+(n-1)\left(l_{1}+l_{2}\right)^{2}\right)^{2}} .
$$

Концентрация напряжений, возникшая на границе полуплоскости $x_{2}=0$, определяется коэффициентом изменчивости среднего квадратического отклонения:

$$
\rho=\frac{\sqrt{D_{11}(0)}}{\sqrt{D_{11}(\infty)}}=\sqrt{1+a_{1}^{2}+a_{2}^{2}+2 a_{1}} .
$$

В таблице 1 приведены значения коэффициента изменчивости $\rho$ в зависимости от степени не-

\begin{tabular}{|c|c|c|c|c|c|c|}
\hline$\frac{h}{n}$ & 0 & 0,25 & 0,5 & 1 & 2 & 4 \\
\hline 1 & 2 & 2 & 2 & 2 & 2 & 2 \\
\hline 3 & 1,22 & 1,46 & 1,68 & 2 & 2,15 & 2,05 \\
\hline 5 & 1 & 1,25 & 1,52 & 2 & 2,30 & 2,09 \\
\hline 7 & 0,89 & 1,13 & 1,42 & 2 & 2,43 & 2,12 \\
\hline 9 & 0,82 & 1,06 & 1,36 & 2 & 2,56 & 2,15 \\
\hline
\end{tabular}
линейности $n$ и параметра нагружения $h=\frac{\sigma_{11}^{0}}{\sigma_{22}^{0}}$. Для упругой полуплоскости величина $\rho$ приведена в работе [2] при коэффициенте Пуассона равным 0,25 и $\sigma_{11}^{0}=\sigma_{22}^{0}$ (соответствует параметру $h=1)$. Она равна 1,55 .

Т а б л и ц а 1

Значения коэффициента изменчивости $\rho$ напряжения $\sigma_{11}$

В таблице 2 приведены в зависимости от переменных $\alpha$ и $n$ значения коэффициента вариации $d_{11}=\frac{\sqrt{D_{11}(0)}}{\sigma_{11}^{0}} 100 \%$ на границе полуплоскости $x_{2}=0$ при $h=2$. При этом значении $h$, как

Т а б л и ц а 2

Значения коэффициента вариации напряжения $\sigma_{11}$ на границе полуплоскости

\begin{tabular}{|l|l|l|l|l|l|}
\hline$\alpha$ & 1 & 3 & 5 & 7 & 9 \\
\hline 0,05 & 5,30 & 4,92 & 4,61 & 4,36 & 4,14 \\
\hline 0,1 & 10,61 & 9,84 & 9,23 & 8,71 & 8,28 \\
\hline 0,2 & 21,21 & 19,69 & 18,45 & 17,42 & 16,55 \\
\hline 0,3 & 31,82 & 29,53 & 27,68 & 26,14 & 24,83 \\
\hline 0,4 & 42,43 & 39,37 & 36,91 & 34,85 & 33,10 \\
\hline 0,5 & 53,03 & 49,22 & 46,13 & 43,56 & 41,38 \\
\hline
\end{tabular}


следует из таблицы 1 , величина $d_{11}$ принимает наибольшее значение (при фиксированных $\alpha$ и $n)$. Как видно из таблицы 2 , для материалов с высоким показателем нелинейности $(n=9)$ коэффициент вариации находится в пределах от $4,14 \%(\alpha=0,05)$ до $41,38 \%(\alpha=0,5)$. В случае низких показателей нелинейности, когда возможна полная физическая линеаризация закона ползучести $(n=1)$ разброс напряжения $\sigma_{11}$ около среднего значения больше: здесь $d_{11}$ заключена в пределах от $5,30 \%$ до $53,03 \%$.

Таким образом, в поверхностном слое флуктуации напряжения $\sigma_{11}$ достигают заметных величин, которые могут быть значительно больше, чем для глубинных слоев.

\section{БИБЛИОГРАФИЧЕСКИЙ СПИСОК}

1. Ломакин В. А. Статистические задачи механики твердых деформируемых тел. - М.: Наука, 1970. - 137c.

2. Ломакин В. А., Шейнин В.И. Концентрация напряжений на границе случайно-неоднородного упругого тела // Изв. АН СССР. МТТ, 1974. — № 2. - С. 124-130.

3. П Подкков B. В., Романов В. А. Концентрация напряжений на границе микронеоднородного упругого полупространства // ПММ, 1978. - Т. 42, Вып. 3. - С. 540-545.

4. Подалков B. В., Романов В. А. Деформация упругого анизотропного микронеоднородного полупространства // ПММ, 1983. - Т. 47, Вып. 3. - С. 455-461.

5. Архипов Н. В. Задача о деформировании микронеоднородного цилиндра // Вестн. МГУ. Сер.1. Математика, механика, 1984. — № 3. - С. 50-54.

6. Кузнецов В. А. Ползучесть стохастически неоднородных сред в условиях плоского напряженного состояния // Математическая физика. — Куйбышев: КпТИ, 1976. - С. 69-74.

7. Попов Н. Н., Самарин Ю. П. Пространственная задача стационарной ползучести стохастически неоднородной среды // ПМТФ, 1985. - № 2. - С. 150-155.

8. Попов Н. Н., Должковой А. А. Нелинейная задача о деформировании стохастически неоднородной плоскости // Математические модели и краевые задачи: Тр. 13 межвуз. конф. Ч. 1. - Самара: СамГТУ, 2003. - С.148-154.

9. Попов Н. Н., Самарин Ю. П. Исследование полей напряжений вблизи границы стохастически неоднородной полуплоскости при ползучести // ПМТФ, 1988. — № 1. - С. 159-164.

10. Вентиель Е.С., Овчаров Л.А. Прикладные задачи теории вероятностей. - М.: Радио и связь, 1983. -416 с.

Поступила 24.12.2005 2.

УдК 539.214; 539.374

Е. П. Кочеров, А. И. Хромов

\section{ДЕФОРМАЦИОННЫЕ СОСТОЯНИЯ И РАЗРУШЕНИЕ ИДЕАЛЬНЫХ ЖЕСТКОПЛАСТИЧЕСКИХ ТЕЛ}

Деформационные состояния идеально жесткопластического тела изображаются точками в пространстве главных деформачий и образуют для несжимаемых тел гиперболическую поверхность третьего порядка. Деформационные процессы изображаются линиями на этой поверхности. Рассматриваются простые проиессы деформирования, когда главные направления тензоров скоростей деформаций и конечных деформаций Альманси совпадают. Показано, что простыле процессы деформирования описывают все возможные непрерывные кривые, лежащие на поверхности деформационных состояний. Вместе с этим непрерывные кривые деформирования отображают и другие сложные прочессы деформирования, связанные с изменением взаимного положения осей тензоров скоростей деформачий и конечных деформаций, при реализации которых требуются различные удельные диссипации энергии. Формулируются деформационно-энергетические критерии разрушения пластических тел с учетом диссипации энергии в процессе деформирования.

1. Основные соотношения. Запишем ассоциированный закон течения

$$
\begin{aligned}
& \varepsilon_{i j}=\lambda \frac{\partial f}{\partial \sigma_{i j}} \text { или } \varepsilon_{i}=\lambda \frac{\partial f}{\partial \sigma_{i}}, \lambda>0, i, j=1,2,3, \\
& \varepsilon_{i j}=\frac{1}{2}\left(V_{i, j}+V_{j, i}\right),
\end{aligned}
$$

где $f\left(\sigma_{i j}\right)$ - функция текучести; $\sigma_{i j}, \varepsilon_{i j}$ —тензоры напряжений и скоростей деформаций; $V_{i}$ - вектор скорости перемещений. 